\title{
PROBABILISTIC TELEPORTATION OF AN ARBITRARY SINGLE-PARTICLE VIA FOUR- PARTICLE ENTANGLED STATE
}

\section{XIAOQING TAN and XU ZHOU}

Department of Mathematics

Jinan University

Guangzhou

Guangdong, 510632

P. R. China

e-mail: ttanxq@jnu.edu.cn

248987644@qq.com

\begin{abstract}
In this paper, we present two different quantum teleportation schemes of an arbitrary single-particle, one is perfect teleportation and the other is probabilistic teleportation. In the first scheme, a four-particle maximally entangled state is used as the quantum channel. Besides, Bob needs to apply appropriate unitary transformations according to Alice's measurement results to recover the unknown state and the success probability is 1 . In the latter scheme, the quantum channel is a four-particle non-maximally entangled state, which is a more general state. Furthermore, Bob needs to introduce an auxiliary qubit so that he can reconstruct the unknown state with the success probability of $2\left(|l|^{2}+|m|^{2}\right)$.
\end{abstract}

2010 Mathematics Subject Classification: 81P40, 81P45, 81P94.

Keywords and phrases: arbitrary single-particle state, quantum teleportation, probabilistic teleportation, appropriate unitary transformations, non-maximally entangled, auxiliary qubit.

Received December 21, 2016

(ㄷ) 2017 Scientific Advances Publishers 


\section{Introduction}

Quantum entanglement, one of the most remarkable features of quantum mechanics, has been used as the fundamental resource of quantum information processing such as quantum teleportation [1], quantum secret sharing [2], quantum computation [3], and quantum secure direct communication [4] and so on. Quantum teleportation, firstly proposed by Bennett et al. [1] in 1993, is the process that a sender (Alice) transmits an unknown quantum state to a distant receiver (Bob) via a quantum channel with the help of some classical information. In 1998, Karlsson and Bourennane [5] presented the first controlled teleportation via GHZ state. In 2009, Nie et al. [6] proposed the non-maximally entangled controlled teleportation using four particles cluster state. Many various teleportation schemes [7-10] of an arbitrary single-particle or a two-particle state have been proposed over the past decades. Moreover, teleportation has been demonstrated with the polarization photon [11] and a single coherent mode of field [12] in optical experiment.

In standard teleportation protocol, Alice performs a Bell-state measurement on the unknown state and one-half of the maximally entangled pair and Bob applies a local unitary operation to recover the unknown state depending on the measurement outcome of Alice. Meanwhile, maximally entangled states, such as Bell state, GHZ state, W state or other multi-particle entangled state, are used as the quantum channel for faithful teleportation. Recently, many teleportation protocols basing on the multi-particle have been proposed [13-15].

Actually, Alice and Bob may not have shared maximally entangled state but some form of non-maximally entangled state (due to some imperfection at the source). If the entangled state used as quantum channel is not maximally entangled, perfect teleportation will not be realized and a finite probability (between 0 to 1 ) will be succeeded instead, which is probabilistic quantum teleportation. 
In this paper, we firstly propose a perfect teleportation scheme of an arbitrary single-particle state via a four-particle maximally entangled state, which is maximally connected and high persistently entangled. And then we a present probabilistic teleportation scheme of an arbitrary single-particle state. The quantum channel is constructed by a fourparticle non-maximally entangled state.

The rest of this article is organized as follows. We propose the prefect teleportation scheme of an arbitrary single-particle state in Section 2 . Section 3 is our main section which will present the probabilistic teleportation of an arbitrary single-particle state via a four-particle nonmaximally entangled state. Finally, we make a brief conclusion in Section 4.

\section{Teleportation of an Arbitrary Single-Particle State}

Suppose Alice (sender) wants to teleport Bob (receiver) an unknown single-particle state, which is

$$
|\gamma\rangle_{a}=(\alpha|0\rangle+\beta|1\rangle)_{a}
$$

where $|\alpha|^{2}+|\beta|^{2}=1$. A four-particle maximally entangled state is used as quantum channel between Alice and Bob, which is the following state:

$$
|\varphi\rangle_{1234}=\frac{1}{2}(|0101\rangle+|0110\rangle+|1001\rangle-|1010\rangle)_{1234} .
$$

Particles $a, 1,2$, and 3 belong to Alice and particle 4 belongs to Bob, respectively. Initially, the joint system before Alice's measurement can be written as follows:

$$
\begin{aligned}
\left|\Psi_{1}\right\rangle_{a 1234}= & |\gamma\rangle_{a} \otimes|\varphi\rangle_{1234} \\
= & (\alpha|0\rangle+\beta|1\rangle)_{a} \otimes \frac{1}{2}(|0101\rangle+|0110\rangle+|1001\rangle-|1010\rangle)_{1234} \\
= & \frac{1}{2}(\alpha|00101\rangle+\alpha|00110\rangle+\alpha|01001\rangle-\alpha|01010\rangle \\
& +\beta|10101\rangle+\beta|10110\rangle+\beta|11001\rangle-\beta|11010\rangle)_{a 1234}
\end{aligned}
$$




$$
\begin{aligned}
= & \frac{1}{2}\left[\left|\phi_{0}\right\rangle_{a 123}(\alpha|0\rangle+\beta|1\rangle)_{4}+\left|\phi_{1}\right\rangle_{a 123}(\alpha|0\rangle-\beta|1\rangle)_{4}\right. \\
& \left.+\left|\phi_{2}\right\rangle_{a 123}(\alpha|1\rangle+\beta|0\rangle)_{4}+\left|\phi_{3}\right\rangle_{a 123}(\alpha|1\rangle-\beta|0\rangle)_{4}\right],
\end{aligned}
$$

where $\left|\phi_{i}\right\rangle_{a 123}(i=0,1,2,3)$ are mutually orthogonal four-particle states given by

$$
\begin{aligned}
& \left|\phi_{0}\right\rangle_{a 123}=\frac{1}{2}(|0011\rangle-|0101\rangle+|1010\rangle+|1100\rangle)_{a_{123}}, \\
& \left|\phi_{1}\right\rangle_{a 123}=\frac{1}{2}(|0011\rangle-|0101\rangle-|1010\rangle-|1100\rangle)_{a_{123}}, \\
& \left|\phi_{2}\right\rangle_{a 123}=\frac{1}{2}(|0010\rangle+|0100\rangle+|1011\rangle-|1101\rangle)_{a_{123}}, \\
& \left|\phi_{3}\right\rangle_{a 123}=\frac{1}{2}(|0010\rangle+|0100\rangle-|1011\rangle+|1101\rangle)_{a_{123}} .
\end{aligned}
$$

In order to realize the teleportation, Alice needs to measure particles $a, 1,2$, and 3 under measurement basis $\left|\phi_{i}\right\rangle_{a 123}(i=0,1,2,3)$. Each measurement result corresponds to a classical double-bit. Alice sends the two classical bits to Bob via a classical channel. Then Bob applies appropriate Pauli operations according to Alice's measurement results to recover the unknown single-particle state. The success probability is 1 . Measurement results of Alice and unitary transformations of Bob are listed in Table 1.

Table 1. The unitary transformations of Bob correspond to Alice's measurement results

\begin{tabular}{|c|c|c|}
\hline $\begin{array}{c}\text { Alice's measurement } \\
\text { results }\end{array}$ & Classical bits & $\begin{array}{c}\text { Bob's unitary } \\
\text { transformations }\end{array}$ \\
\hline$\left|\phi_{0}\right\rangle_{a 123}$ & 00 & $I$ \\
\hline$\left|\phi_{1}\right\rangle_{a 123}$ & 01 & $\sigma_{z}$ \\
\hline$\left|\phi_{2}\right\rangle_{a 123}$ & 10 & $\sigma_{x}$ \\
\hline$\left|\phi_{3}\right\rangle_{\alpha 123}$ & 11 & $i \sigma_{y}$ \\
\hline
\end{tabular}




\section{Probabilistic Teleportation of an Arbitrary Single-Particle State}

In this section, Alice also teleports the unknown single particle state

$$
|\gamma\rangle_{a}=(\alpha|0\rangle+\beta|1\rangle)_{a}
$$

to Bob, and they take a four-particle non-maximally entangled state as the quantum channel

$$
|\psi\rangle_{1234}=(j|0101\rangle+k|0110\rangle+l|1001\rangle-m|1010\rangle)_{1234},
$$

where $|j|^{2}+|k|^{2}+|l|^{2}+|m|^{2}=1$ and $|j|>|k|>|l|>|m|$. Particles $a, 1,2$, and 3 belong to Alice and particle 4 belongs to Bob, respectively. Initially, the joint system before Alice's measurement can be written as follows:

$$
\begin{aligned}
\left|\psi_{2}\right\rangle_{a 1234}= & |\gamma\rangle_{a} \otimes|\psi\rangle_{1234} \\
= & (\alpha|0\rangle+\beta|1\rangle)_{a} \otimes(j|0101\rangle+k|0110\rangle+l|1001\rangle-m|1010\rangle)_{1234} \\
= & (\alpha j|00101\rangle+\alpha k|00110\rangle+\alpha l|01001\rangle-\alpha m|01010\rangle \\
& +\beta j|10101\rangle+\beta k|10110\rangle+\beta l|11001\rangle-\beta m|11010\rangle)_{a 1234} \\
= & \frac{1}{\sqrt{2}}\left[\left|\delta_{0}\right\rangle_{a 123}(\alpha k|0\rangle+\beta l|1\rangle)_{4}+\left|\delta_{1}\right\rangle_{a 123}(\alpha k|0\rangle-\beta l|1\rangle)_{4}\right. \\
& +\left|\delta_{2}\right\rangle_{a 123}(\alpha l|1\rangle+\beta k|0\rangle)_{4}+\left|\delta_{3}\right\rangle_{a 123}(\alpha l|1\rangle-\beta k|0\rangle)_{4} \\
& +\left|\delta_{4}\right\rangle_{a 123}(\alpha m|0\rangle+\beta j|1\rangle)_{4}+\left|\delta_{5}\right\rangle_{a 123}(\alpha m|0\rangle-\beta j|1\rangle)_{4} \\
& \left.+\left|\delta_{6}\right\rangle_{a 123}(\alpha j|1\rangle+\beta m|0\rangle)_{4}+\left|\delta_{7}\right\rangle_{a 123}(\alpha j|1\rangle-\beta m|0\rangle)_{4}\right]
\end{aligned}
$$

where $\left|\delta_{i}\right\rangle_{a 123}(i=0,1, \ldots, 7)$ are mutually orthogonal four-particle states given by

$$
\begin{aligned}
& \left|\delta_{0}\right\rangle_{a 123}=\frac{1}{\sqrt{2}}(|0011\rangle+|1100\rangle)_{a 123}, \\
& \left|\delta_{1}\right\rangle_{a 123}=\frac{1}{\sqrt{2}}(|0011\rangle-|1100\rangle)_{a 123},
\end{aligned}
$$




$$
\begin{aligned}
& \left|\delta_{2}\right\rangle_{a 123}=\frac{1}{\sqrt{2}}(|0100\rangle+|1011\rangle)_{a 123}, \\
& \left|\delta_{3}\right\rangle_{a 123}=\frac{1}{\sqrt{2}}(|0100\rangle-|1011\rangle)_{a 123}, \\
& \left|\delta_{4}\right\rangle_{a 123}=\frac{1}{\sqrt{2}}(-|0101\rangle+|1010\rangle)_{a 123}, \\
& \left|\delta_{5}\right\rangle_{a 123}=\frac{1}{\sqrt{2}}(-|0101\rangle-|1010\rangle)_{a 123}, \\
& \left|\delta_{6}\right\rangle_{a 123}=\frac{1}{\sqrt{2}}(|0010\rangle-|1101\rangle)_{a 123}, \\
& \left|\delta_{7}\right\rangle_{a 123}=\frac{1}{\sqrt{2}}(|0010\rangle+|1101\rangle)_{a 123} .
\end{aligned}
$$

Next, Alice needs to measure particles $a, 1,2$, and 3 under measurement basis $\left|\delta_{i}\right\rangle_{a 123}(i=0,1, \ldots, 7)$. Each measurement result corresponds to a classical three-bit. Alice sends the three classical bits to Bob via a classical channel. Then Bob applies appropriate Pauli operations according to Alice's measurement results and then the state $|\gamma\rangle_{4}$ will collapse to $|\gamma\rangle_{4}^{i}(i=1,2,3,4)$. The unitary transformations of Bob correspond to Alice's measurement results and the collapsed states under the unitary transformations are listed in Table 2. 
Table 2. The unitary transformations of Bob correspond to Alice's measurement results and the collapsed states under the unitary transformations

\begin{tabular}{|c|c|c|c|}
\hline $\begin{array}{l}\text { Alice's } \\
\text { measurement } \\
\text { results }\end{array}$ & $\begin{array}{c}\text { Classical } \\
\text { bits }\end{array}$ & $\begin{array}{l}\text { Bob's unitary } \\
\text { transformations }\end{array}$ & $\begin{array}{c}\text { The collapsed states under } \\
\text { the unitary } \\
\text { transformations }\end{array}$ \\
\hline$\left|\delta_{0}\right\rangle_{a 123}$ & 000 & $I$ & \multirow{2}{*}{$|\gamma\rangle_{4}^{1}=\frac{1}{\sqrt{2}}(\alpha k|0\rangle+\beta l|1\rangle)_{4}$} \\
\hline$\left|\delta_{1}\right\rangle_{a 123}$ & 001 & $\sigma_{z}$ & \\
\hline$\left|\delta_{2}\right\rangle_{a 123}$ & 010 & $\sigma_{x}$ & \multirow{2}{*}{$|\gamma\rangle_{4}^{2}=\frac{1}{\sqrt{2}}(\alpha l|0\rangle+\beta k|1\rangle)_{4}$} \\
\hline$\left|\delta_{3}\right\rangle_{a 123}$ & 011 & $i \sigma_{y}$ & \\
\hline$\left|\delta_{4}\right\rangle_{a 123}$ & 100 & $I$ & \multirow{2}{*}{$|\gamma\rangle_{4}^{3}=\frac{1}{\sqrt{2}}(\alpha m|0\rangle+\beta j|1\rangle)_{4}$} \\
\hline$\left|\delta_{5}\right\rangle_{a 123}$ & 101 & $\sigma_{z}$ & \\
\hline$\left|\delta_{6}\right\rangle_{\alpha 123}$ & 110 & $\sigma_{x}$ & \multirow{2}{*}{$|\gamma\rangle_{4}^{4}=\frac{1}{\sqrt{2}}(\alpha j|0\rangle+\beta m|1\rangle)_{4}$} \\
\hline$\left|\delta_{7}\right\rangle_{a 123}$ & 111 & $i \sigma_{y}$ & \\
\hline
\end{tabular}

Suppose Bob gets the state

$$
|\gamma\rangle_{4}^{1}=\frac{1}{\sqrt{2}}(\alpha k|0\rangle+\beta l|1\rangle)_{4}
$$

In order to acquire the initial state, Bob needs to introduce an auxiliary qubit with the original state $|0\rangle_{A}$ under the basis $\left\{|00\rangle_{A 4},|01\rangle_{A 4}\right.$, $\left.|10\rangle_{A 4},|11\rangle_{A 4}\right\}$. A collective unitary transformation $U_{1}$ is made, where

$$
\begin{gathered}
U_{1}=\left(\begin{array}{cc}
A_{1} & A_{2} \\
A_{2} & -A_{1}
\end{array}\right), \\
A_{1}=\operatorname{diag}\left(a_{0}, a_{1}\right), \quad A_{2}=\operatorname{diag}\left(\sqrt{1-a_{0}^{2}}, \sqrt{1-a_{1}^{2}}\right), \\
\left(a_{0}, a_{1}\right)=\left(\frac{l}{k}, 1\right),
\end{gathered}
$$




$$
U_{1}=\left(\begin{array}{cccc}
\frac{l}{k} & 0 & \sqrt{1-\left(\frac{l}{k}\right)^{2}} & 0 \\
0 & 1 & 0 & 0 \\
\sqrt{1-\left(\frac{l}{k}\right)^{2}} & 0 & -\frac{l}{k} & 0 \\
0 & 0 & 0 & -1
\end{array}\right) .
$$

For example,

$$
\begin{aligned}
U_{1}|00\rangle & =a_{0}|00\rangle+\sqrt{1-a_{0}^{2}}|10\rangle \\
& =\frac{l}{k}|00\rangle+\sqrt{1-\left(\frac{l}{k}\right)^{2}}|10\rangle .
\end{aligned}
$$

Then with the unitary transformation $U_{1}$ operation, the unnormalized state $|0\rangle_{A} \otimes|\gamma\rangle_{4}^{1}$ will be changed as follows:

$$
\begin{aligned}
U_{1}\left(|0\rangle_{A} \otimes|\gamma\rangle_{4}^{1}\right) & =U_{1}\left[\frac{1}{\sqrt{2}}(\alpha k|00\rangle+\beta l|01\rangle)\right]_{A 4} \\
& =\frac{\alpha k}{\sqrt{2}}\left(\frac{l}{k}|00\rangle_{A 4}+\sqrt{1-\left(\frac{l}{k}\right)}|10\rangle_{A 4}\right)+\frac{\beta l}{\sqrt{2}}|01\rangle_{A 4} \\
& =|0\rangle_{A} \otimes \frac{l}{\sqrt{2}}(\alpha|0\rangle+\beta|1\rangle)_{4}+|1\rangle_{A} \otimes \frac{1}{\sqrt{2}}\left(\alpha k \sqrt{1-\left(\frac{l}{k}\right)^{2}}\right)|0\rangle_{4} .
\end{aligned}
$$

Equation (9) is also unnormalized, so Bob needs to measure the auxiliary particle under measurement basis $\{|0\rangle,|1\rangle\}$. The result $|1\rangle_{A}$ means the failed teleportation, while the result $|0\rangle_{A}$ means the successful case. Thus, Bob will acquire the initial single-particle state

$$
|\gamma\rangle_{a}=(\alpha|0\rangle+\beta|1\rangle)_{a}
$$

with the success probability $\frac{|l|^{2}}{2}$. 
Similarly, the other possible states can be discussed in the same way. While the values of $a_{i}(i=0,1)$ in the unitary transformation $U_{1}$ are different and described in Table 3. Synthesizing all cases (8 kinds in all), the probability of successful teleportation is $4\left(\frac{|l|^{2}}{2}+\frac{|m|^{2}}{2}\right)=2\left(|l|^{2}+|m|^{2}\right)$.

Table 3. The values of $a_{i}(i=0,1)$ in the unitary transformation $U_{1}$

\begin{tabular}{|c|c|c|c|}
\hline $\begin{array}{c}\text { The state of the } \\
\text { particle 4 }\end{array}$ & $a_{0}$ & $a_{1}$ & $\begin{array}{c}\text { The probability } \\
\text { of the successful } \\
\text { teleportation }\end{array}$ \\
\hline$|\gamma\rangle_{4}^{1}$ & $\frac{l}{k}$ & 1 & $\frac{|l|^{2}}{2}$ \\
\hline$|\gamma\rangle_{4}^{2}$ & 1 & $\frac{l}{k}$ & $\frac{||^{2}}{2}$ \\
\hline$|\gamma\rangle_{4}^{3}$ & 1 & $\frac{m}{j}$ & $\frac{|m|^{2}}{2}$ \\
\hline$|\gamma\rangle_{4}^{4}$ & $\frac{m}{j}$ & 1 & $\frac{|m|^{2}}{2}$ \\
\hline
\end{tabular}

\section{Conclusion}

In this paper, we propose two different quantum teleportation schemes of an arbitrary single-particle state. In the first scheme, a fourparticle maximally entangled state is used as quantum channel between Alice and Bob. Furthermore, Bob can easily reconstruct the original state by applying appropriate unitary transformations according to Alice's measurement results and the success probability is 1 . In the second scheme, Alice also teleports this arbitrary single-particle state via a fourparticle non-maximally entangled state, which is probabilistic teleportation. In the second scheme, Bob needs to introduce an auxiliary qubit to recover the initial state and the success probability is $2\left(|l|^{2}+|m|^{2}\right)$. At last, the latter has an obvious superiority that the quantum channel is a more general state. 


\section{Acknowledgements}

The research is funded by National Natural Science Foundation of China, under Grant Nos. 61672014 and 61502200, and Natural Science Foundation of Guangdong Province, China, under Grant Nos. 2016A030313090 and 2014A030310245, and Science and Technology Planning Project of Guangdong Province, China, under Grant No. 2013B010401018.

\section{References}

[1] C. H. Bennett et al., Teleportation an unknown quantum state via dual classical and Einstein-Podolsky-Rosen channels, Phys. Rev. Lett. 70 (1993), 1895.

[2] L. Liu, C. Tsai and T. Hwang, Quantum secret sharing using symmetric w state, Int. J. Theor. Phys. 51(7) (2012), 2291-2306.

[3] M. A. Neilsen and I. L. Chuang, Quantum Computation and Quantum Information, New York: Cambridge University Press, (2000), 171-211.

[4] G. Gao, Bidirectional quantum secure communication based on one-dimensional four-particle cluster states, Int. J. Theor. Phys. 53(7) (2014), 2282-2287.

[5] A. Karlsson and M. Bourennane, Quantum teleportation using three-particle entanglement, Phys. Rev. A 58(6) (1998), 4394-4400.

[6] Y. Nie, Z. Hong, Y. Huang, X. Yi and S. Li, Non-maximally entangled controlled teleportation using four particles cluster states, Int. J. Theor. Phys. 48(5) (2009), 1485-1490.

[7] P. Agrawal and A. Pati, Perfect teleportation and superdense coding with w states, Phys. Rev. A 74(6) (2006), 062320.

[8] S. Sheng, Splitting quantum information via w state, Phys. Rev. A 74(5) (2006), 054303 .

[9] D. Li and Z. Cao, Teleportation of two-particle entangled state via cluster state, Commun. Theor. Phys. 47(3) (2007), 464-466.

[10] M. Sreraman and K. Prasanta, Prefect teleportation, quantum-state sharing, and superdense coding through a genuinely entangled five-qubit state, Phys. Rev. A 77(3) (2008), 032321.

[11] D. Bouwmeester et al., Experimental quantum teleportation, Nature 390 (1997), 575.

[12] A. Furusawa et al., Unconditional quantum teleportation, Science 282 (1998), 706.

[13] M. Zhong and Z. Lin, Quantum teleportation of a three-qubit state using a five-qubit cluster state, Int. J. Theor. Phys. 53(12) (2014), 4079-4082. 
[14] J. Liu, Y. Li and Y. Nie, Controlled teleportation of an arbitrary two-particle pure or mixed state by using a five-qubit cluster state, Int. J. Theor. Phys. 49(8) (2010), 1976-1984.

[15] Z. Man, Y. Xia and N. An, Genuine multiqubit entanglement and controlled teleportation, Phys. Rev. A 75(5) (2007), 052306. 